\title{
Effect of Interfacial Interaction between Glass-Fiber and Matrix Nylon-6 on Nonlinear Dynamic Viscoelasticity and Fatigue Behavior for Glass-Fiber Reinforced Nylon-6
}

\author{
Shintaro Komatsu, Atsushi TAKAHARA*, and Tisato KAJIYAMA ${ }^{\dagger}$ \\ Department of Applied Chemistry, Graduate School of Engineering, Kyushu University, 6-10-1 Hakozaki, \\ Higashi-ku, Fukuoka 812-8581, Japan \\ *Institute for Fundamental Research of Organic Chemistry, Kyushu University, 6-10-1 Hakozaki, \\ Higashi-ku, Fukuoka 812-8581, Japan
}

(Received July 18, 2002; Accepted October 7, 2002)

\begin{abstract}
Effect of (glass-fiber/matrix nylon-6) interfacial interactions on fatigue behaviors of glass-fiber reinforced nylon-6 (GF/Ny6) under a strain controlled condition was investigated on the basis of in situ nonlinear dynamic viscoelasticity. Ny6s mixed with surface-modified and -unmodified short glass-fibers (SMSGF and SUSGF) were used as specimens. An extent of nonlinearity of dynamic viscoelasticity was expressed by nonlinear viscoelastic parameter, NVP, which was calculated from coefficients of the Fourier expanded series of the response signal during a fatigue cycle. The nonlinear dynamic viscoelasticity for both (GF/Ny6)s became remarkable with the progress of fatigue damage. The magnitude of NVP for the (SUSGF/Ny6) was always greater than that for the (SMSGF/Ny6) at a given fatigue time. Since the interface between SUSGF and Ny6 was peeled off during the fatigue test due to the weak interaction, the fatigue damage easily occurred for the (SUSGF/Ny6). Such leads to the non-uniform propagation of the imposed strain through the (SUSGF/Ny6), resulting in an increase of NVP. In the case of the (SMSGF/Ny6), the fatigue damage slowly progressed due to the strong interfacial interaction. Consequently, the nonlinearity of the dynamic viscoelasticity for the (SMSGF/Ny6) was depressed in comparison with the (SUSGF/Ny6).

KEY WORDS Fatigue Behavior / Short Glass-Fiber Reinforced Nylon-6 / Interfacial Interaction / Nonlinear Viscoelastic Parameter /
\end{abstract}

Polymer composites are a class of materials that polymer matrix is reinforced by fillers such as glassfibers or carbon-fibers with a small amount. Recently, polymer composites have been widely used for structural components of transports and constructions as substitutes of metal materials due to their comparatively high specific strength and modulus. When polymer composites are applied to the above-mentioned purpose, it is important to understand the fatigue mechanisms, and to predict the fatigue lifetime. This is because the fatigue fracture of them could cause fatal accidents.

Fatigue mechanisms of fiber-reinforced polymers have been discussed on the basis of scanning electron microscopy (SEM) observations of fractured surfaces. ${ }^{1,2}$ In general, a fatigue damage of polymer composites starts from debonding at fiber ends. Then, the debonding progresses along the (fiber / polymer) interface, and eventually, polymer composites are fractured. In the case of weak interfacial interaction, debonding easily progresses even at the initial stage of fatigue cycles. On the contrary, interfacial debonding is hard to occur for composites with strong interfacial interaction and thus an imposed load effectively transfers from matrix polymer to fibers, resulting in an improve- ment of fatigue strength. ${ }^{1,3-7}$ Although SEM is an useful apparatus, fatigue tests must be interrupted to observe sample surfaces. Therefore, it seems difficult by SEM observation to follow how fatigue damages such as interfacial debonding progress in a specimen. In order to clarify the fatigue fracture mechanisms for polymer composites, it is necessary to propose a parameter which can quantitatively monitor the fatigue progress.

Tanaka et al. studied the nonlinear viscoelastic behavior for high-density polyethylene (HDPE) under large cyclic deformation on the basis of simultaneous measurements of dynamic viscoelasticity and dynamic birefringence. ${ }^{8}$ They claimed that the nonlinear viscoelasticity was associated with the local fracture of higher-order structure of HDPE. Kajiyama et al. proposed nonlinear viscoelastic parameter, NVP, which can quantitatively investigate the nonlinearity of dynamic viscoelasticity for solid polymers during the fatigue process..$^{6,7,9-14}$ They showed that an increase in NVP related to the irreversible structural changes of the polymers. $^{9-14}$ Also, the fatigue mechanism for glassfiber reinforced nylon-66 under a stress controlled fatigue test was similarly discussed, and it was concluded that the irreversible structural changes such as debond-

${ }^{\dagger}$ To whom correspondence should be addressed (Tel : +81-92-642-3560, E-mail: kajiyama@cstf.kyushu-u.ac.jp). 


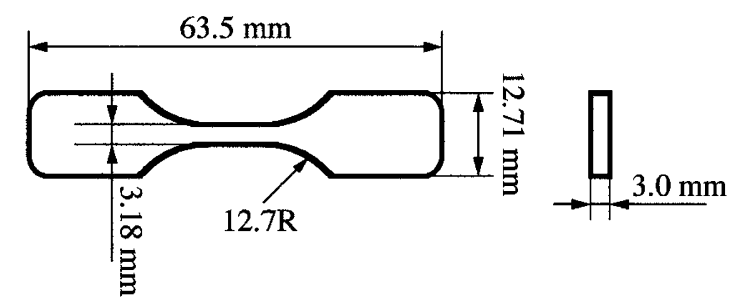

Figure 1. Dimensions of specimen for fatigue test.

ing and microcrack growth remarkably related to the nonlinear viscoelasticity. ${ }^{7}$

The purpose of this study is to evaluate the effect of interfacial interaction on fatigue behaviors for short glass-fiber reinforced nylon-6 (GF/Ny6) by measuring the magnitude of NVP. Then, the fatigue mechanism of the (GF/Ny6) was discussed.

\section{EXPERIMENTAL}

\section{Materials and Dynamic Viscoelastic Measurement}

The specimens used in this study were short glassfiber reinforced nylon-6 (GF/Ny6), which has been widely applied as structural components. Matrix polymer of Nylon-6 (UBE Nylon 1015B, $M_{\mathrm{w}}=15000$ ) was kindly supplied from Ube Industries, Ltd. Surfacemodified short glass-fibers (SMSGF) which were treated with aminosilane coupling agent and surfaceunmodified ones (SUSGF) were used as reinforcing components. Diameter and average length of glassfibers were $13 \mu \mathrm{m}$ and $c a$. $400 \mu \mathrm{m}$, respectively. The fraction of glass-fibers in the (GF/Ny6)s was fixed to be $30 \mathrm{wt} \%$. Figure 1 illustrates the schematic representation of an ASTM-D1822 Type L tension-impact specimen for the fatigue test. The test pieces of the (GF/Ny6)s was prepared by injection molding. The gauge length of the dumb-bell shape specimens was $20 \mathrm{~mm}$. By SEM observation of cross-section of the (GF/Ny6), the glass-fibers almost oriented along the direction of molding, that is, the longitudinal direction of the specimens.

In order to evaluate thermal molecular motions of the nylon- 6 chains around glass-fibers, the temperature dependences of dynamic viscoelastic properties for the (GF/Ny6)s and the nylon-6 homopolymer were obtained with Rheovibron DDV-01FP (A\&D Co., Ltd.) at the frequency of $11 \mathrm{~Hz}$, in the temperature range from $120 \mathrm{~K}$ to $440 \mathrm{~K}$ under dried nitrogen gas purge. The heating rate was $1 \mathrm{~K} \mathrm{~min}^{-1}$.

\section{Evaluation of Fatigue Behavior for (GF/Ny6)s Based on Nonlinear Dynamic Viscoelastic Analysis}

The fatigue behaviors for the (GF/Ny6)s were evaluated by using a homemade fatigue tester which can

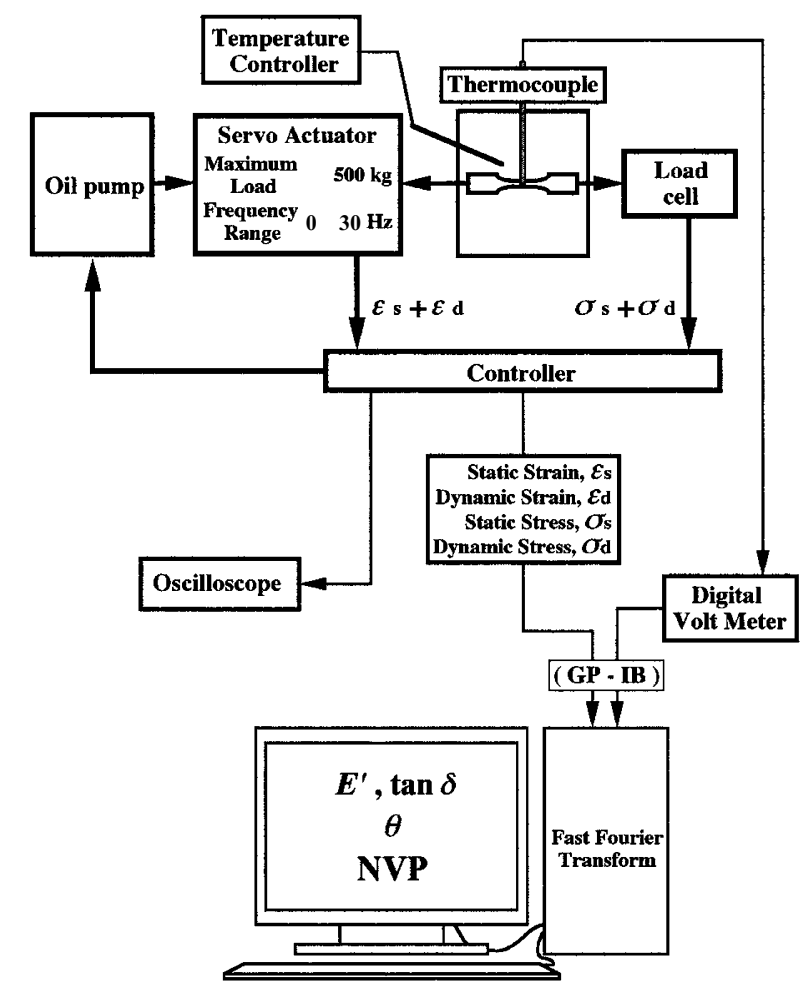

Figure 2. Block diagram of fatigue tester.

monitor nonlinearity of dynamic viscoelasticity during cyclic straining. ${ }^{6,9-14}$ Figure 2 shows the blockdiagram of the fatigue tester. Strain and stress signals were collected by a personal computer with GP-IB interface. The degree of nonlinear dynamic viscoelasticity was continuously analyzed by nonlinear viscoelastic parameter, NVP being defined as follows. When a sinusoidal strain is imposed to specimens with an angular frequency, $\omega$, the responded stress, $\sigma(t)$ can be expanded by Fourier series given by eq 1 .

$$
\begin{aligned}
\sigma(t)= & \sigma_{\mathrm{s}}+\sigma_{1} \sin \left(\omega t+\delta_{1}\right)+\sigma_{2} \sin \left(2 \omega t+\delta_{2}\right)+\cdots \\
& +\sigma_{\mathrm{n}} \sin \left(n \omega t+\delta_{\mathrm{n}}\right)
\end{aligned}
$$

where $\sigma_{\mathrm{s}}, \sigma_{1}$, and $\delta_{1}$ are the static stress, fundamental stress amplitude and phase difference angle, respectively. $\sigma_{2}, \sigma_{3}, \ldots$, and $\delta_{2}, \delta_{3}, \ldots$, are the higher harmonic stress amplitudes and higher harmonic phase difference angles, respectively. Assuming that the degree of nonlinear dynamic viscoelasticity can be represented by the contribution of higher-order terms, NVP can be defined by eq 2. ${ }^{6,9-14}$

$$
N V P=\frac{\sigma_{2}+\sigma_{3}+\cdots+\sigma_{10}}{\sigma_{1}}
$$

Where the number of higher harmonics, $n$, was taken up to 10 in our study because the $\sigma_{\mathrm{n}}$ for $n>11$ were negligibly small. The magnitude of NVP increases with the increasing nonlinearity of dynamic viscoelasticity. ${ }^{6,7,9-14}$

The frequency of fatigue tests was $11 \mathrm{~Hz}$ and the ambient temperature was $303 \mathrm{~K}$. The condition of fatigue 


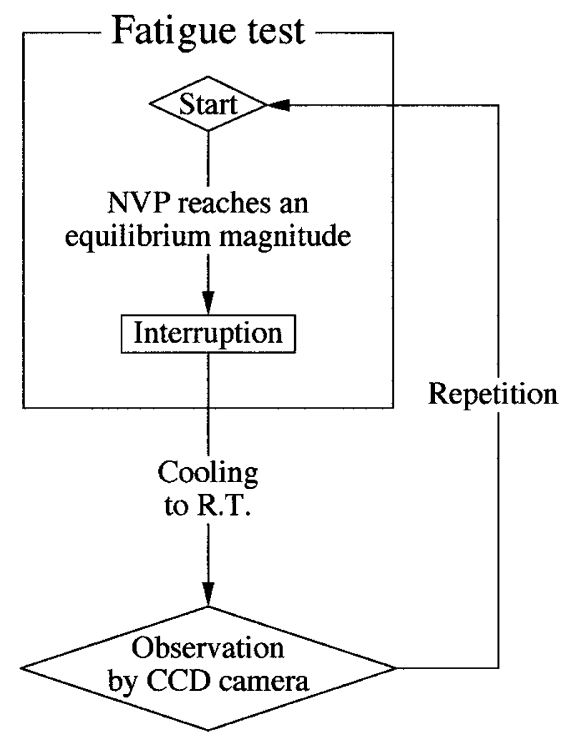

Figure 3. Flow chart of the repetition test during the initial fatigue process.

tests was tension-compression cyclic straining in dried nitrogen atmosphere. Surface temperature rise, $\theta(=$ $T_{\mathrm{s}}-T_{0}$, ) was continuously monitored during cyclic fatigue, where $T_{\mathrm{s}}$ and $T_{0}$ were temperatures of specimen surface and the environment, respectively. The surface temperature was continuously measured at the center of specimen with an adhesive type thermocouple.

In order to discuss the relationship between the magnitude of NVP and fatigue damage of the (GF/Ny6)s, the tensile properties of the (GF/Ny6)s being subjected to various fatigue cycles were measured at $303 \mathrm{~K}$ with the constant strain rate of $1.0 \mathrm{~mm} \mathrm{~min}^{-1}$.

\section{Repetition Tests for (GF/Ny6)s in an Initial Fatigue Process}

To reveal the relationship between the degree of nonlinear dynamic viscoelasticity and morphological changes for the (GF/Ny6)s, repetition tests in an initial fatigue process were carried out. Figure 3 shows the flow chart of this repetition test. First, when a magnitude of NVP reached an equilibrium value, the fatigue test was intermitted. Then, the surface morphology of specimen was observed by using a charge-coupled imaging device (CCD) camera. These operations were repeated until the specimen was fractured.

\section{SEM Observation of (GF/Ny6)s Morphology}

Morphology of fatigued fracture surface and crosssection of the (GF/Ny6)s were observed by SEM (S2150, Hitachi Ltd.). After a magnitude of NVP of the (GF/Ny6)s reached a given value, fatigue tests were interrupted. Then, the cross-section of the $(\mathrm{GF} / \mathrm{Ny} 6) \mathrm{s}$ was obtained by microtoming the (GF/Ny6)s. The fractured surface and the cross-section of the (GF/Ny6)s were

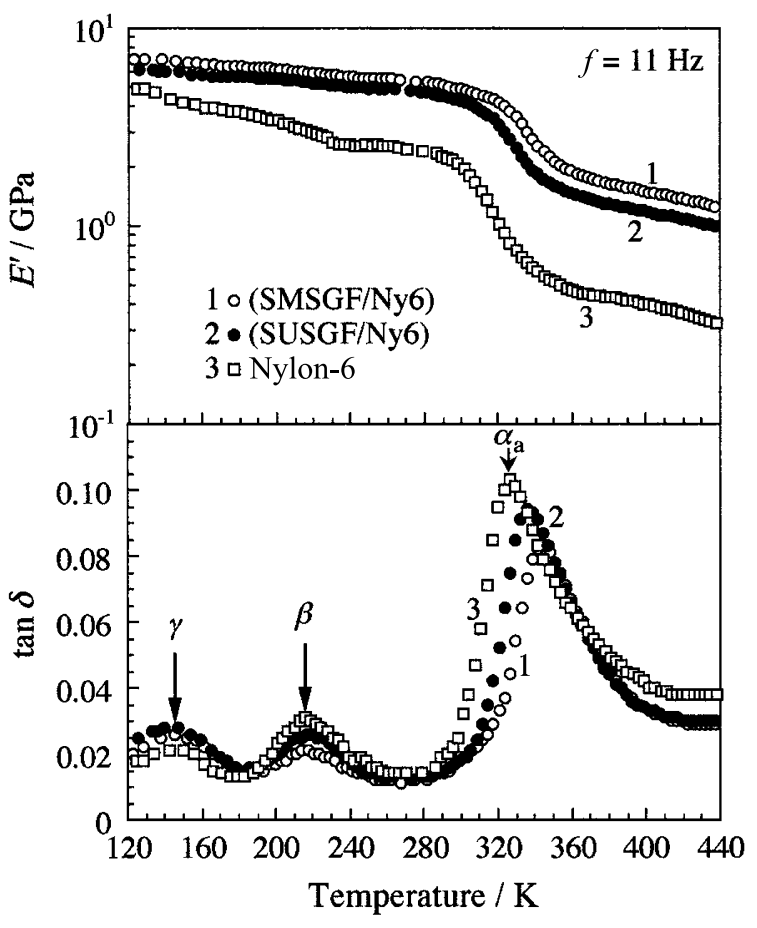

Figure 4. Temperature dependences of $E^{\prime}$ and $\tan \delta$ for the (GF/Ny6)s and the nylon-6 homopolymer at $11 \mathrm{~Hz}$.

coated with gold to avoid charging of the specimen during SEM observation.

\section{RESULTS AND DISCUSSION}

\section{Linear Viscoelastic Properties of (GF/Ny6)s}

Figure 4 shows the temperature dependences of dynamic storage modulus, $E^{\prime}$ and loss tangent, tan $\delta$ measured in a linear viscoelastic regime for the (SMSGF/Ny6), the (SUSGF/Ny6) and the nylon-6 homopolymer. In the temperature ranges employed, the magnitude of $E^{\prime}$ was decreased in the order of the (SMSGF/Ny6), the (SUSGF/Ny6) and the nylon-6 homopolymer. The temperature dependence of $\tan \delta$ exhibited three mechanical absorptions around $330 \mathrm{~K}$, $220 \mathrm{~K}$, and $150 \mathrm{~K}$, respectively. The absorption observed at $330 \mathrm{~K}$ corresponds to the $\alpha_{\mathrm{a}}$-absorption being characteristics of micro-Brownian motion in amorphous phases of nylon-6. ${ }^{15-17}$ The absorption observed at $220 \mathrm{~K}$ is the $\beta$-absorption being attributed to segments containing amide groups not involved in the formation of hydrogen bonds. ${ }^{17-19}$ The $\gamma$-absorption observed around $150 \mathrm{~K}$ is attributed to the motion of methylene parts of nylon-6 chains. ${ }^{15,17-20}$ The peak temperature of $\alpha_{\mathrm{a}}$-absorption increased in the order of the nylon-6 homopolymer, the (SUSGF/Ny6) and the (SMSGF/Ny6). It is clear that the thermal molecular motion of nylon- 6 segments in the amorphous regions was more restricted at the interface region between SMSGF and matrix nylon-6 of the (SMSGF/Ny6) in 


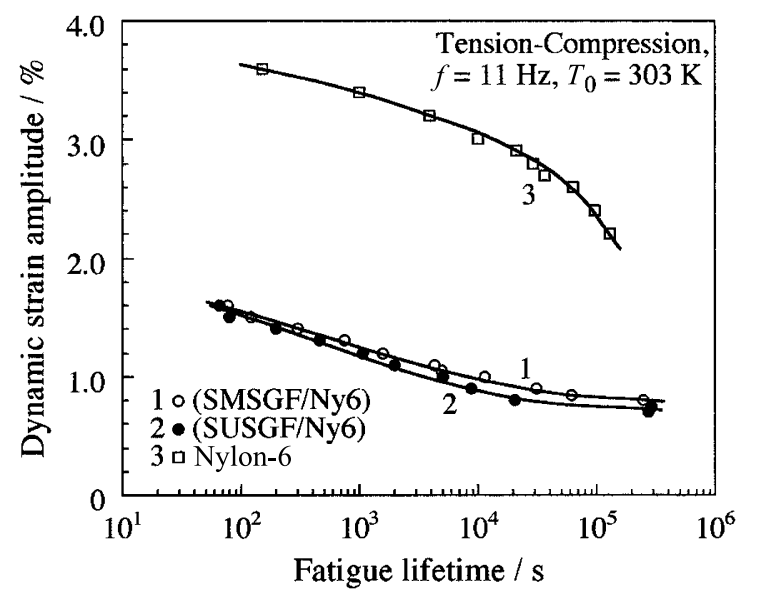

Figure 5. Relationship between imposed strain amplitudes and fatigue lifetime for the (GF/Ny6)s and the nylon-6 homopolymer under cyclic straining at $11 \mathrm{~Hz}$ and $303 \mathrm{~K}$.

comparison with that of the (SUSGF/Ny6). Therefore, it is reasonable to consider that the interfacial interaction between SMSGF and matrix nylon- 6 in the (SMSGF/Ny6) was more strengthened by the aminosilane treatment of glass-fiber surface. Then, it is expected that the interfacial interaction between glassfibers and matrix nylon-6 influences fatigue behaviors of the (SMSGF/Ny6) and the (SUSGF/Ny6).

\section{Effect of Interfacial Interaction on Fatigue Lifetime for (GF/Ny6)s}

The effect of interfacial interaction between glassfibers and matrix nylon- 6 on fatigue characteristics for the (GF/Ny6)s was examined. Figure 5 shows the relationships between fatigue lifetime and imposed strain amplitude for the (SMSGF/Ny6), the (SUSGF/Ny6) and the nylon- 6 homopolymer. The responded stress of the nylon- 6 homopolymer was much smaller than those of the (GF/Ny6)s at given strain amplitudes because of the low modulus of the nylon- 6 . Therefore, the fatigue lifetime of the nylon- 6 homopolymer remarkably increased in comparison with those of the (GF/Ny6)s under strain-controlled fatigue tests. At large strain amplitudes, the fatigue lifetime of the (SMSGF/Ny6) was almost comparable to that of the (SUSGF/Ny6). When large strain amplitudes were imposed on the (GF/Ny6)s, surface temperatures of both (SMSGF/Ny6) and (SUSGF/Ny6) remarkably increased much more than the glass transition temperature $\left(T_{\mathrm{g}}\right)$ of matrix nylon- 6 . Therefore, the ductile type of fatigue fracture was accelerated for the $(\mathrm{GF} /$ Ny6)s. ${ }^{21,22}$ On the other hand, the fatigue lifetime of the (SMSGF/Ny6) increased in comparison with that of the (SUSGF/Ny6) as small strain amplitudes were applied. It is reasonable to consider that the interfacial interaction between glass-fibers and matrix nylon- 6 in-

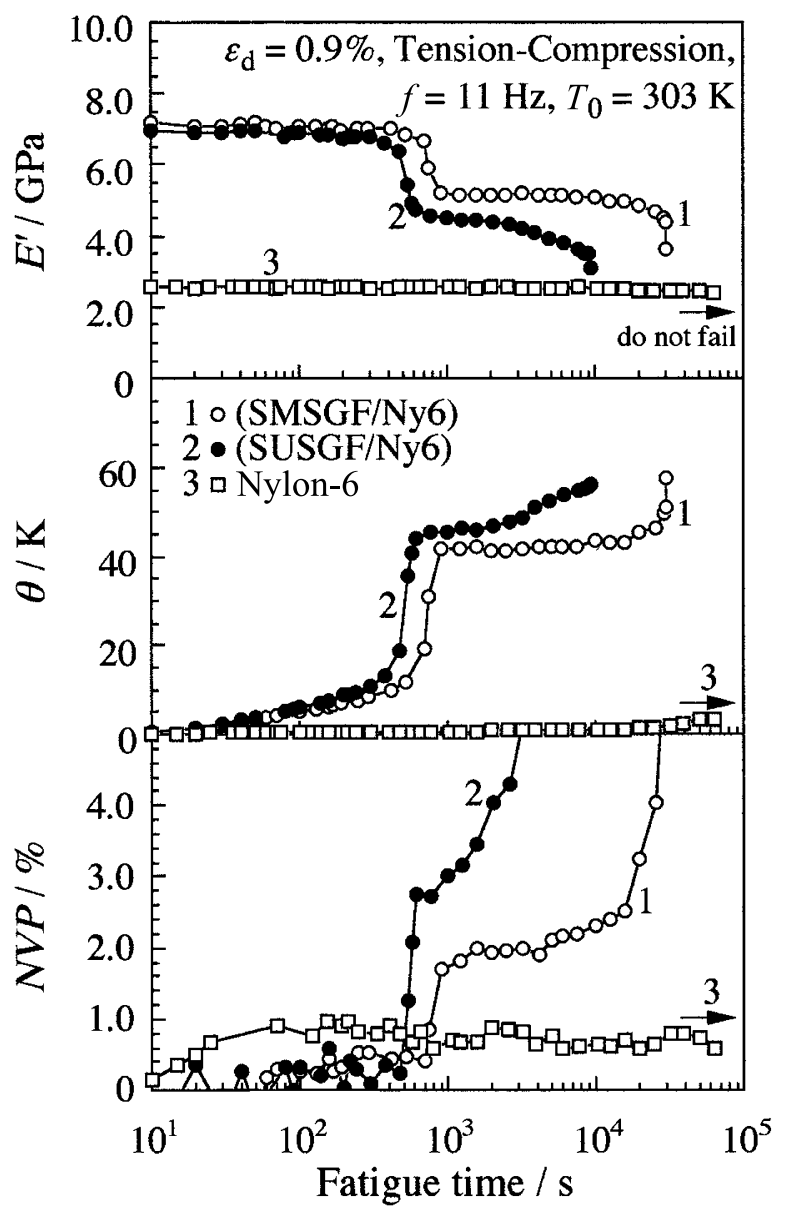

Figure 6. Variations of $E^{\prime}, \theta$, and NVP for the (GF/Ny6)s and the nylon- 6 homopolymer during the fatigue process under the imposed strain amplitude of $0.9 \%$ at $11 \mathrm{~Hz}$ and $303 \mathrm{~K}$.

fluences the fatigue lifetime of the (GF/Ny6)s at small strain amplitudes. The effect of interfacial interaction on the fatigue behaviors will be discussed in the following section.

Fatigue Behavior Analysis for (GF/Ny6)s Based on Nonlinear Dynamic Viscoelastic Measurement

When small strain amplitudes were imposed on the (GF/Ny6)s, the difference in fatigue lifetime between (SMSGF/Ny6) and (SUSGF/Ny6) increased, as shown in Figure 5. The fatigue behaviors for the (GF/Ny6)s at small strain amplitudes were evaluated on the basis of nonlinear dynamic viscoelastic measurement.

Figure 6 shows the fatigue time dependences of $E^{\prime}, \theta$, and NVP for the (SMSGF/Ny6), the (SUSGF/Ny6) and the nylon- 6 homopolymer at the small strain amplitude of $0.9 \%$. The nylon- 6 homopolymer was not fractured within the time range of this measurement. The magnitudes of $E^{\prime}, \theta$, and NVP for the nylon-6 homopolymer were almost constant during the fatigue process. On the other hand, those for the (GF/Ny6)s remarkably changed with a distinct decrease or increase at a fatigue time. In the initial fatigue process, initial stage, 
Table I. Tensile properties for the (GF/Ny6)s after cyclic fatigue under dynamic strain amplitude of $0.9 \%$ at $11 \mathrm{~Hz}$ and $303 \mathrm{~K}$

\begin{tabular}{lccccc}
\hline & $\begin{array}{c}\text { Fatigue time } \\
(\mathrm{s})\end{array}$ & $\begin{array}{c}\text { NVP } \\
(\%)\end{array}$ & $\begin{array}{c}\text { Tensile strength } \\
(\mathrm{MPa})\end{array}$ & $\begin{array}{c}\text { Modulus } \\
(\mathrm{GPa})\end{array}$ & $\begin{array}{c}\text { Yield strain } \\
(\%)\end{array}$ \\
\hline (SMSGF/Ny6) & - & - & 163.0 & 7.50 & 3.53 \\
& 600 & 1.81 & 141.9 & 7.16 & 3.34 \\
& 17000 & 3.86 & 115.6 & 7.00 & 2.36 \\
(SUSGF/Ny6) & - & - & 148.1 & 7.18 & 3.21 \\
& 400 & 1.93 & 122.3 & 6.90 & 2.90 \\
& 800 & 3.61 & 117.4 & 6.83 & 2.74 \\
& 6000 & 9.23 & 81.7 & 6.56 & 1.69 \\
\hline
\end{tabular}

the magnitudes of NVP for the (GF/Ny6)s slightly increased accompanying a decrease in $E^{\prime}$ and a rise of $\theta$. At critical fatigue time, the magnitudes of NVP remarkably increased and then, reached certain values. The region, in which magnitudes of $E^{\prime}, \theta$, and NVP were almost constant during the fatigue process, is defined as equilibrium stage. After passing through the equilibrium stage, the magnitudes of NVP and $\theta$ again started to increase monotonically, and also, the magnitude of $E^{\prime}$ simultaneously decreased. Finally, the (GF/Ny6)s were fractured accompanying remarkable changes in $E^{\prime}, \theta$, and NVP.

To clarify an origin of an increase in NVP, tensile properties for the $(\mathrm{GF} / \mathrm{Ny} 6) \mathrm{s}$ were evaluated. Table I shows the tensile properties for the (GF/Ny6)s at various magnitudes of NVP. The magnitude of $E^{\prime}$ in Figure 6 decreased in comparison with the modulus in Table I due to the effect of an increase in $\theta$ during the fatigue process. The regions that the magnitudes of NVP reached $1.81 \%$ and $3.86 \%$ for the (SMSGF/Ny6) were in the equilibrium stage and in the stage just before the final fracture, respectively. The magnitudes of NVP of $1.93 \%, 3.61 \%$, and $9.23 \%$ for the (SUSGF/Ny6) were in the initial stage and in the stages after the equilibrium stage and just before the final fracture, respectively. The tensile strength, the modulus and the yield strain for both (SMSGF/Ny6) and (SUSGF/Ny6) decreased with increasing magnitudes of NVP. Therefore, Table I indicates that an increase in NVP corresponds to a progress of fatigue damage. This means that a magnitude of NVP is one of the measures or the parameters to evaluate a progress of fatigue damage of the (GF/Ny6)s. Once the fatigue time goes beyond the equilibrium stage, the magnitudes of NVP again started to increase monotonically, meaning that fatigue damages might be progressed.

The initial stage of fatigue process for the (SMSGF/Ny6) as indicated by the initial slight change of NVP was longer than that of the (SUSGF/Ny6). Kajiyama et al. revealed that debonding at interfaces between glass-fibers and matrix nylon-66 occurred before the specimen temperature rose under a stress-controlled fatigue test. ${ }^{7}$ Hertzberg and Manson implied that an incubation (initial) stage was associated with an initial material state involving a few mobile defects and a state wherein such defects were generated at a slow rate. ${ }^{23}$ Because defects such as interfacial debonding in the (GF/Ny6)s occurred, the magnitudes of NVP for the (GF/Ny6)s slightly increased in the initial stage. Therefore, the period of initial stage for the (SMSGF/Ny6) was longer than that for the (SUSGF/Ny6), since it seems difficult to occur interfacial debonding between SMSGF and matrix nylon- 6 for the (SMSGF/Ny6) due to the strong interfacial interaction.

The period of equilibrium stage as shown by the second flat region of $E^{\prime}$ of the (SMSGF/Ny6) markedly prolonged in comparison with that of the (SUSGF/Ny6). The degree of $\theta$ in the equilibrium stage for the (SUSGF/Ny6) was larger than that for the (SMSGF/Ny6). This may be attributed to a larger net strain amplitude imposed only on matrix nylon6 of the (SUSGF/Ny6) compared with that of the (SMSGF/Ny6) since cyclic deformation of matrix polymer causes a rise of $\theta$. In the case of the (GF/Ny6)s, the occurrence of nonlinear dynamic viscoelastic property during a fatigue process is mainly originated from debonding at interface and plastic deformation of matrix nylon-6. Since the interfacial interaction of the (SUSGF/Ny6) was weak, it seems that irreversible structural changes such as debonding in the (SUSGF/Ny6) occur easily. Therefore, the distribution of responded stress amplitude in the (SUSGF/Ny6) was less uniform than that in the (SMSGF/Ny6) and then, the magnitude of NVP for the (SUSGF/Ny6) was larger than that for the (SMSGF/Ny6) in the equilibrium stage. The areas where the irreversible structural changes occurred during a fatigue process of the (GF/Ny6)s were observed by CCD camera and SEM as describe in the next section.

Relationship between Nonlinear Dynamic Viscoelasticity and Morphological Changes for (GF/Ny6)s

The magnitude of NVP for the (SMSGF/Ny6) decreased in comparison with that for the (SUSGF/Ny6) 
at the given strain amplitude as shown in Figure 6. The irreversible structural changes such as debonding at interface between glass-fibers and matrix nylon- 6 occurred during the fatigue processes for the (GF/Ny6)s. The areas of irreversible structural changes in the (GF/Ny6)s which reached the various magnitudes of NVP were observed by means of CCD camera and SEM. The flow chart of test method is shown in Figure 3. Figure 7 shows the relationship between the repeating numbers of the repetition test, $\mathrm{N}$ and the magnitudes of NVP at the end of initial stage for the (GF/Ny6)s. Since both (SMSGF/Ny6) and (SUSGF/Ny6) were fractured after some repeat-

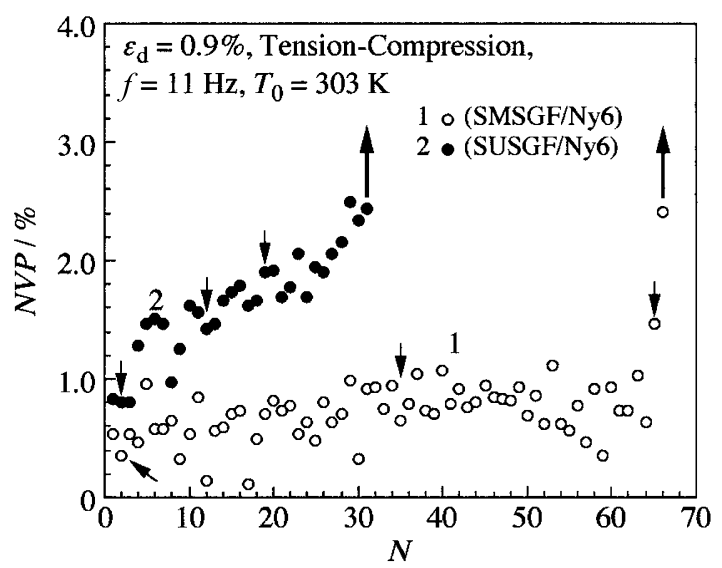

Figure 7. Variations of NVP for the (GF/Ny6)s during the repetition test under the imposed strain amplitude of $0.9 \%$ at $11 \mathrm{~Hz}$ and $303 \mathrm{~K}$.

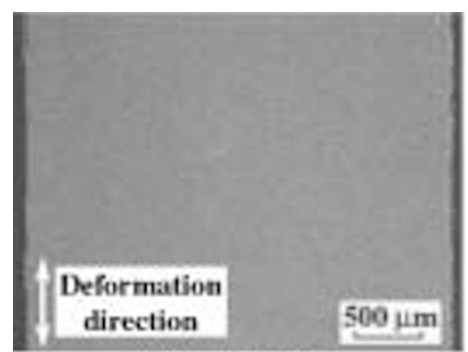

(a) 1st test

$(\mathrm{NVP}=1.94 \%)$

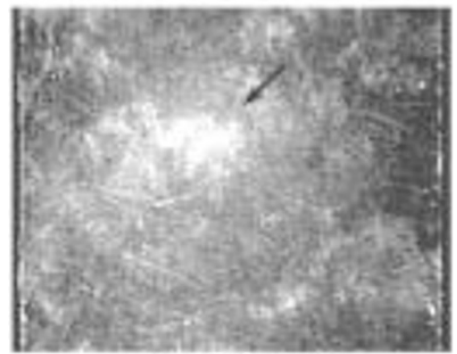

(c) 65th test

$(\mathrm{NVP}=6.44 \%)$ ing numbers, it is clear that fatigue damages in the (GF/Ny6)s progressed during the initial stage. The periods of 1st repeating number of the repetition test $(\mathrm{N}$ $=1)$ for the $(\mathrm{SMSGF} / \mathrm{Ny} 6)$ and the (SUSGF/Ny6) were ca. $600 \mathrm{~s}$ and $400 \mathrm{~s}$, respectively. Then, each period after the 2nd $(\mathrm{N}>1)$ was $c a .150 \mathrm{~s}$ and $90 \mathrm{~s}$, respectively. Since irreversible structural changes such as interfacial debonding already occurred in the (GF/Ny6)s during 1 st repeating number, each period after the 2 nd decreased in comparison with that of 1 st. The magnitudes of NVP for the (SMSGF/Ny6) slightly increased and then, remarkably increased at the last several repeating numbers. In the case of the (SUSGF/Ny6), the magnitudes of NVP increased monotonically from early repeating numbers. In order to reveal the relationship between these changes of NVP values and the structural changes of the (GF/Ny6)s, the surface morphology of the (GF/Ny6)s after each repeating number corresponding to the $\mathrm{N}$ shown by arrows in Figure 7 was observed by CCD camera. Figure 8 shows the CCD images of the (SMSGF/Ny6) surface after various repeating numbers of the repetition test. With an increase in repeating numbers, stress whitening began to occur at a certain area of the (SMSGF/Ny6) surface as indicated by an arrow. The plastic deformation spread from the initial position and grew as cracks with an increase in repeating numbers and then, the (SMSGF/Ny6) was fractured. The magnitude of NVP of the (SMSGF/Ny6) increased with an increase in the area of plastic deforma-

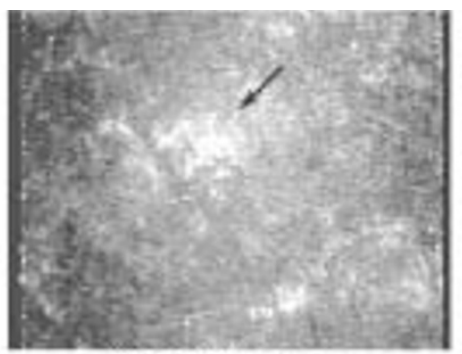

(b) 35th test

$(\mathrm{NVP}=3.49 \%)$

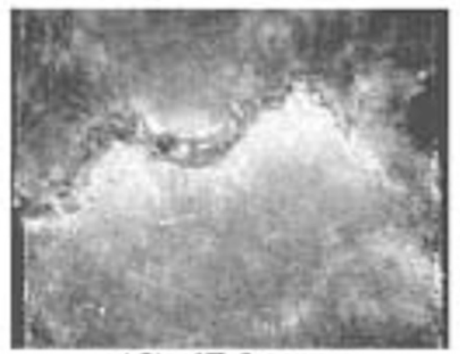

(d) 67 th test

(Failure of the specimen)

Figure 8. Optical micrographs for the (SMSGF/Ny6) surface with various magnitudes of NVP during the repetition test at $11 \mathrm{~Hz}$ and $303 \mathrm{~K}$. 


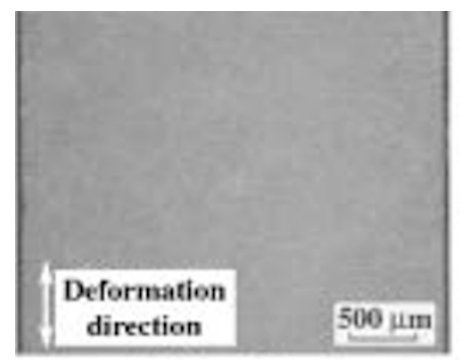

(a) 2nd test

(NVP $=3.35 \%$ )

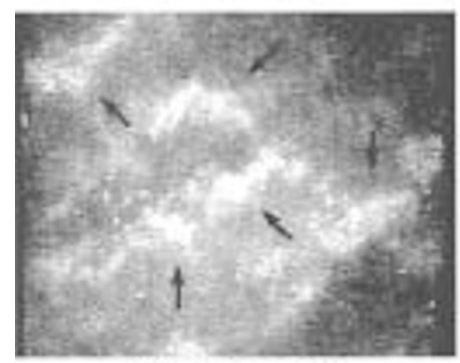

(c) 19th test

$(\mathrm{NVP}=8.54 \%)$

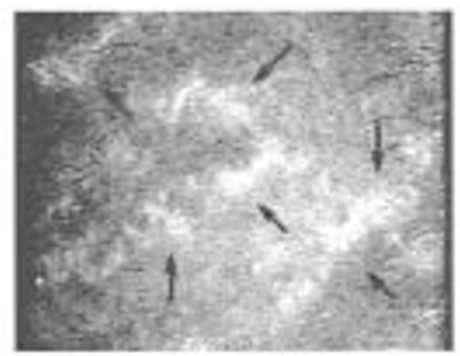

(b) 12th test

$(\mathrm{NVP}=7.14 \%)$

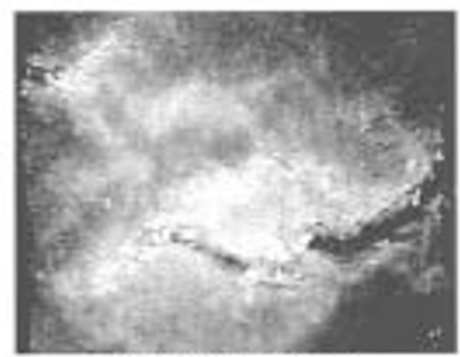

(d) 32th test

(Failure of the specimen)

Figure 9. Optical micrographs for the (SUSGF/Ny6) surface with various magnitudes of NVP during the repetition test at $11 \mathrm{~Hz}$ and $303 \mathrm{~K}$.

tion. When the plastic deformation occurred, the distribution of responded stress around the area became nonuniform. Therefore, the degree of nonlinearity of dynamic viscoelasticity of the (GF/Ny6)s increased with an increase in the areas of plastic deformation. Figure 9 shows the CCD images of the (SUSGF/Ny6) surface after various repeating numbers of the repetition test. At early repeating numbers, plastic deformation occurred at several areas of the (SUSGF/Ny6) surface as indicated by arrows. With an increase in the repeating numbers, each area of plastic deformation spread from their initial positions and connected each other, and finally, the (SUSGF/Ny6) was fractured. The magnitude of NVP of the (SUSGF/Ny6) also increased with an increase in the areas of plastic deformation. It is reasonable to consider that since plastic deformation was easily occurred in the (SUSGF/Ny6), the magnitude of NVP of the (SUSGF/Ny6) increased in comparison with that of the (SMSGF/Ny6).

In order to discuss the plastic deformation in the (GF/Ny6)s in detail, the internal morphology of the (GF/Ny6)s was observed by using SEM. Figure 10 shows the internal morphology of the (GF/Ny6)s before fatigue test and after being subjected to the cyclic fatigue until the magnitudes of NVP for the (SMSGF/Ny6) and the (SUSGF/Ny6) reached 6.35\% and $7.25 \%$, respectively. The directions of cyclic deformation and glass-fibers orientation in SEM photographs are horizontal. As shown in Figure 10a, debonding at the interface between SMSGF and matrix nylon-6 in the (SMSGF/Ny6) was not observed before a fatigue test. In the case of the (SUSGF/Ny6), debonding at the interface between SUSGF and matrix nylon- 6 was observed even before a fatigue test as shown in Figure 10b. When the (SUSGF/Ny6) is injection-molded, interfacial debonding may occur due to weak interfacial interaction. After the $(\mathrm{GF} / \mathrm{Ny} 6) \mathrm{s}$ were subjected to cyclic fatigue, debonding at the interface along glass-fiber surface and remarkable plastic deformation at glass-fiber ends in both (SMSGF/Ny6) and (SUSGF/Ny6) were observed as shown in Figures $10 \mathrm{c}$ and $10 \mathrm{~d}$. Therefore, an extent of stress whitening observed by using CCD camera (Figures 8 and 9) corresponded to the area of debonding and plastic deformation around glass-fibers. It became clear that when the plastic deformation of matrix nylon- 6 around glass-fibers in the (GF/Ny6)s occurred during a fatigue process, the magnitude of NVP increased. Besides, the magnitude of NVP for the (SUSGF/Ny6) increased in comparison with that for the (SMSGF/Ny6), because the plastic deformation around glass-fibers easily occurred in the (SUSGF/Ny6).

\section{CONCLUSIONS}

The fatigue behaviors for the (GF/Ny6)s were evaluated on the basis of nonlinear dynamic viscoelastic measurement. Nonlinear viscoelastic parameter, 


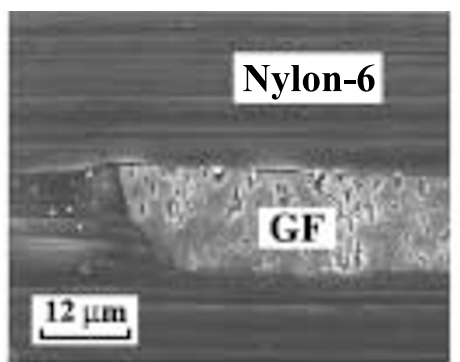

(a) Before the fatigue test for (SMSGF/Ny6)

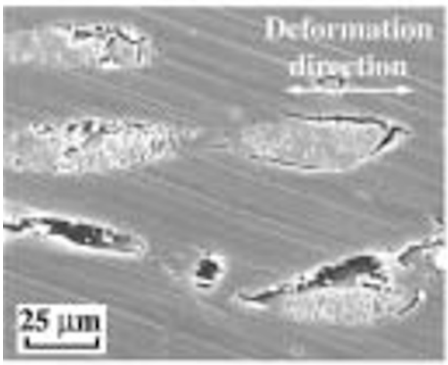

(c) After the fatigue test for (SMSGF/Ny6) $\mathrm{NVP}=6.35 \%$

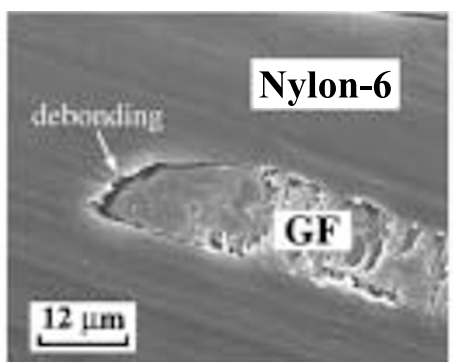

(b) Before the fatigue test for (SUSGF/Ny6)

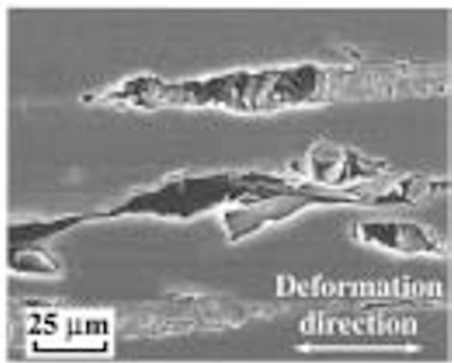

(d) After the fatigue test for (SUSGF/Ny6) $\mathrm{NVP}=7.25 \%$

Figure 10. SEM photographs of internal morphology for the (GF/Ny6)s before fatigue test and after being subjected to the cyclic fatigue until the magnitudes of NVP were 6.35\% (SMSGF/Ny6) and 7.25\% (SUSGF/Ny6) at $11 \mathrm{~Hz}$ and $303 \mathrm{~K}$.

NVP increased with the progress of fatigue damage of the (GF/Ny6)s. The magnitude of NVP for the (SMSGF/Ny6) with strong interfacial interaction was comparatively small because of the slow accumulation of fatigue damage. On the other hand, the magnitude of NVP for the (SUSGF/Ny6) with the weak interfacial interaction increased in comparison with that for the (SMSGF/Ny6) since the interfacial debonding easily occurred and spread. It became clear that progress of fatigue damage of the (GF/Ny6)s can be monitored by NVP.

Acknowledgments. The authors thank Mr. Kazuya Noda (Asahi Chemical Industry Co., Ltd.) for the preparation of specimens and helpful discussions.

\section{REFERENCES}

1. R-W. Lang, J-A. Manson, and R-W. Hertzberg, J. Mater. Sci., 22, 4015 (1987).

2. J-J. Horst and J-L. Spoormaker, J. Mater. Sci., 32, 3641 (1997).

3. A. Takahara, T. Magome, and T. Kajiyama, J. Polym. Sci., Polym. Phys. Ed., 32, 839 (1994).

4. A. Yamashita, S. Higashi, S. Komatsu, A. Takahara, and T. Kajiyama, Key Eng. Mater., 137, 147 (1998).

5. A. Yamashita, A. Takahara, and T. Kajiyama, Compos. Interface, 6, 247 (1999).
6. S. Komatsu, A. Yamashita, A. Takahara, and T. Kajiyama, Rept. Progr. Polym. Phys. Jpn., 42, 327 (1999).

7. K. Noda, A. Takahara, and T. Kajiyama, Polymer, 42, 5803 (2001).

8. A. Tanaka, M. Fukuda, H. Nagai, M. Shinohara, and S. Onogi, J. Polym. Sci., Polym. Phys. Ed., 27, 2283 (1989).

9. N-J. Jo, A. Takahara, and T. Kajiyama, Polym. J., 25, 721 (1993).

10. N-J. Jo, A. Takahara, and T. Kajiyama, Polym. J., 26, 1024 (1994).

11. T. Liang, K. Tokunaga, A. Yamashita, A. Takahara, and K. Kajiyama, Polym. Bull., 36, 477 (1996).

12. T. Liang, A. Takahara, and T. Kajiyama, Polym. J., 28, 801 (1996).

13. N-J. Jo, A. Takahara, and T. Kajiyama, Polymer, 38, 5195 (1997).

14. T. Liang, A. Takahara, K. Saito, and T. Kajiyama, Polymer, 39, 5387 (1998).

15. J. Rubin, and R-D. Andrews, Polym. Eng. Sci., 8, 302 (1968).

16. A. Anton, J. Appl. Polym. Sci., 12, 2117 (1968).

17. V. Frosini, and E. Butta, J. Polym. Sci., B, 9, 253 (1971).

18. T. Kawaguchi, J. Appl. Polym. Sci., 4, 56 (1959).

19. J. Kolarik and J. Janacek, J. Polym. Sci., C, 16, 441 (1967).

20. R-D. Andrews, and T-J. Hammack, J. Polym. Sci., B, 3, 659 (1965).

21. A. Takahara, K. Yamada, T. Kajiyama, and M. Takayanagi, $J$. Appl. Polym. Sci., 25, 597 (1980).

22. A. Takahara, K. Yamada, T. Kajiyama, and M. Takayanagi, J. Appl. Polym. Sci., 26, 1085 (1981).

23. R-W. Hertzberg, and J-A. Manson, "Fatigue of engineering plastics", Academic Press, Inc., New York, N.Y., 1980. 